\title{
Magnetic Bearing Proposal Design for a General Unbalanced Rotor System enhanced because of using sensors/actuators based in nanostructures
}

\author{
Jesus A. Calderón ${ }^{1,2,}$, Eliseo B. Barriga ${ }^{2}$, Roland $\mathrm{Mas}^{2}$, Luis Chirinos ${ }^{2}$,Enrique Barrantes ${ }^{2}$, Jorge Alencastre $^{2}$, Julio . . \\ Tafur $^{2}$, Oscar Melgarejo ${ }^{2}$, John H. Lozano ${ }^{2,3}$, Bjorn Heinrich ${ }^{1,2}$, Enrique Aguilar ${ }^{2}$, Juan C. Lengua ${ }^{2}$ \\ ${ }^{1}$ TU Ilmenau University of Technology, Institute of Physics and IMN MacroNano, Germany \\ ${ }^{2}$ Pontificia Universidad Católica del Perú Energy Laboratory and Mechatronic Departament, Perú \\ ${ }^{3}$ Northen (Artic) Federal University named after MV. Lomonosov, Arkhangelsk, Russia.
}

\begin{abstract}
Rotor systems need bearings in order to keep uniformity of rotational movement transmission. However, bearingsgenerate friction and energy losses due to heating transmisssion through the friction; for this reason, mechanicak bearings are replaced by magnetic bearings owing to avoid energy losing because of friction. We designed Active Magnetic Bearings (AMB) to transmit rotational movement from source of movement (motor) through the rotor to the movement receptor (such as a conveyor belt). Magnetic Bearings need accuracy during System Identification process and a sophisticated control algorithm to get an uniform rotation movement transmission. In this work also it was analyzed and proved by simulations that Active Magnetic Bearings composed with sensors /actuators based in nanostructures are faster and robust compared with AMB based in traditional sensors/actuators. It because, nanostructures receive and send signals better way tan traditional sensors/actuators, because of high oredered nanoarrays improve sensor/actuator properties.
\end{abstract}

\section{Introduction}

There are many machines that transmit movement through rotation of their shaft, and this causes disturbances such as intense vibrations, noise and heat transmission, and wear, problems that would unbalance the rotor. Vibrations can be avoided with passive mechanisms such as dampers around their holders, obtaining a balanced transmission of the rotor, but noise, heat transmission and wear would continue. These are the problems caused by the use of mechanical bearings, used in industries. The following solutions are proposed, which is an active magnetic bearing system, to compensate for the problems obtained by mechanical bearings, in the same way to compensate the unbalance, in the same way to obtain a uniform motion transmission with different frequency values [1],[2],[3].

There are three types of systems for centring shafts that work with magnetism, which are: passive magnetic bearings that are implemented only with ferromagnetic or neodymium magnets that produce a magnetic force, active magnetic bearings that operate by means of electromagnetic coils that obtain magnetic force by means of electrical energy, and hybrid magnetic cushions that are the union of passive and active magnetic bearings. The use of each is proposed according to the system that is required. "Active magnetic bearing system (AMB)", by means of magnetism they centre an axis towards a theoretical axis proposed by the author, using nanosensors and nano-actuators it would be obtained answers in short time to comparison of the common sensors and actuators, by means of correct control strategies, plus a classic hardware and with new software strategies, it would be obtained a performance of the magnetic bearings to comparison of the mechanical bearings due to the solids and wide range of work. When generalizing our system, difficulties are encountered such as describing the complex dynamics of each proposed rotor, describing its systems and reducing them to practical models through adaptive/predictive coefficients in the main mathematical model. In addition, we suggest some pragmatic hardware designs such as, for example, in electromagnets for fixing the rotor.

\section{Rotor scheme analysis}

Magnetic Bearing Systems are usually applied to replace mechanical bearings in rotor systems of machines such as chillers (refrigeration), turbines, compressors systems; so, its mathematical needs to know total forces applied in the rotor. Hence, it is represented through figure 1 a general rotor scheme in Cartesian coordinates X, Y, Z, in which is generalized " $n$ " hybrid electromagnet that (through right control) can compensate all forces in order to get stability and balance while rotor is under movement.

Corresponding author: alan.calderon@ @ucp.edu.pe 


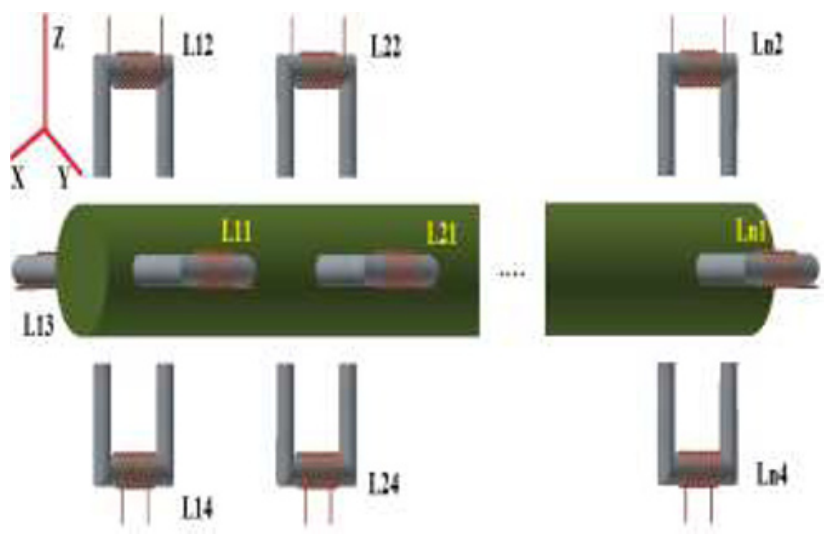

Fig. 1. General rotor (shaft) scheme under equilibrium of forces for $\mathrm{AMB}$ analysis.

For this reason, in figure 2 is shown the "Resultant Force" which is obtained by equilibrium analysis from gravity forcé (depicted by $\mathrm{Fg}$ ) with magnetic forces (depicted by FIL11, FIL12, FIL13, FIL14 until FILn1, FILn2, FILn3 , FILn4, it because every current from hybrid electromagnets IL11, IL12, IL13, IL14 until ILn1, ILn2, ILn3, ILn4) as it is for AMB systems with magnetic bearings over the rotor ends, also it is analized reactions because of contact rotor support (holders) that is represented by FR, furthermore, the resultant force due to rotation rotor movement FC. However, while it is necessary to find a balance on rotors which are fixed over one of its ends, it because of the motor that it is connected, then it must to be analyzed the reaction among them, it means FR may be the reaction while motor DC is on the end $\mathrm{X} 0, \mathrm{Y} 0, \mathrm{Z} 0$, in this context the resultant force must to be so carefully calculated due to for unbalanced rotor the equilibrium does not depends of Force balance by Newton Second law only, it depends of torque analysis too. Therefore, by this criterion it can be obtained the balance analysis in the rotor system.

When movement is transferred by the rotor, whether it is under different values of high frequencies (it was considered for simulations in this work "High frequencies" values bigger than 3200 RPM), then it must to be studied own rotor deformation which increase the unbalance in the system. Nevertheless, in this work is analyzed unbalance not because own response during movement transmission, that axis is represented by line " $E$ " in figure 2.

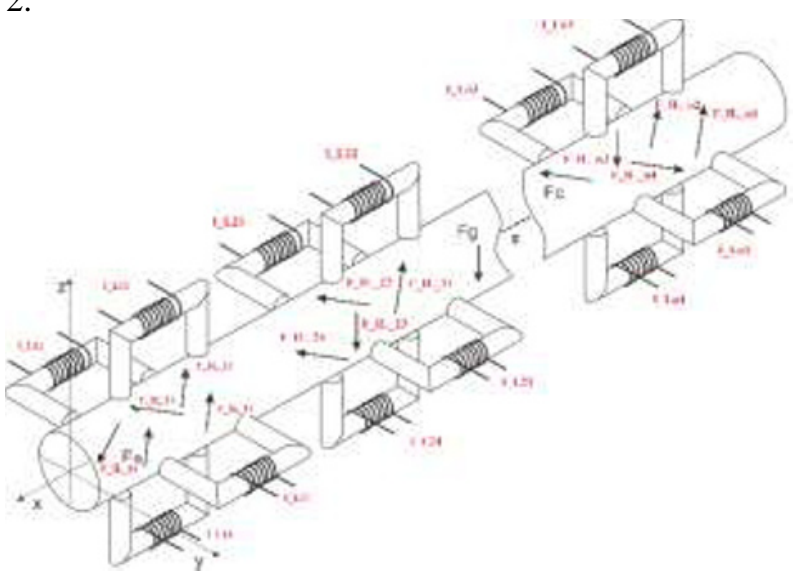

Fig. 2. Total, forces applied on the rotor (shaft).
In order to get a mathematical model to achieve or calculate physical parameters more close to the real design, so there were analyzed second Newton law for circular and linear equilibrium and dynamic, as a consequence there were expected physical parameters and geometrical parameters with criterion which can be enhanced while getting system identification after to design the prototype.

There are many machinery that needs AMB such as a combustion machine (It is shown in figure 3), which use mechanical bearing to reduce imbalances caused through vibrations during operating work of these machines. Nevertheless, mechanical bearing generates heating around machine's rotor, which is a big disadvantage to not achieve good performance of them.

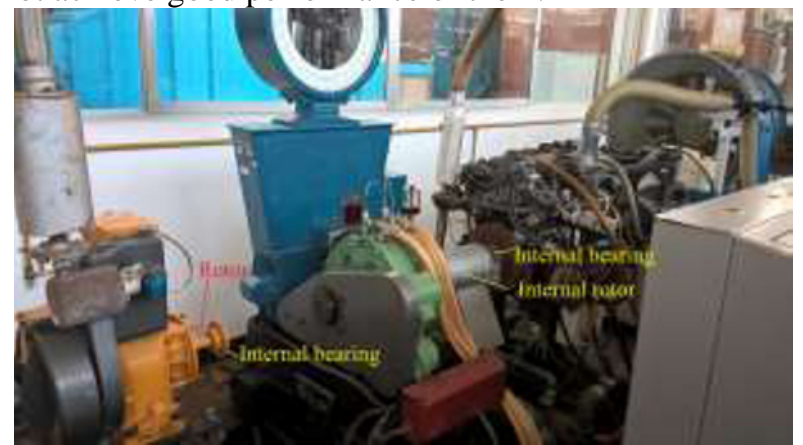

Figure 3. Movement transmission through combustion machine's rotor of "Energy Laboratory Pontifiicia Universidad Católica del Perú”.

It is necessary to recognize the problem before to look for a theoretical understanding of the system; for this reason in this work was analyzed a prototype, that is shown in figure 4, which has a DC motor with a fixed rotor to an unbalanced shaft. Furthermore, owing to measure imbalance movement transmission, it is used position sensors [7]; by other side, due to create a force to achieve the balance in rotor movement, a magnetic force is produced through an electromagnetic actuators (electromagnets) of course it can be produced by simple magnets too. Nevertheless, getting control is achieved by electromagnets that is known as "Active mechanisms".

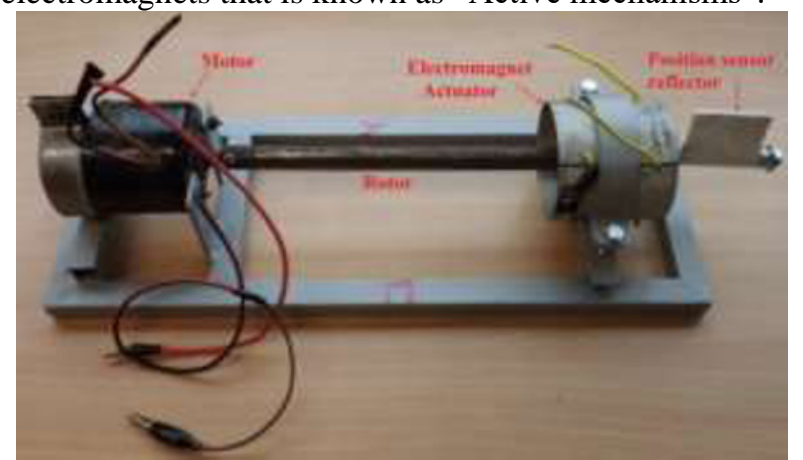

Figure 4. Balanced rotor system prototype by strategies based on AMB.

Furthermore, because of knowledge and experience obtained getting control for first prototype showed above, in figure 2 is depicted another prototype in design, for that is joined one more electromagnet actuator in order to verify generalized mathematical models developed in this 
research, for which also was proposed hybrid mechanisms to achieve desired magnetic force.

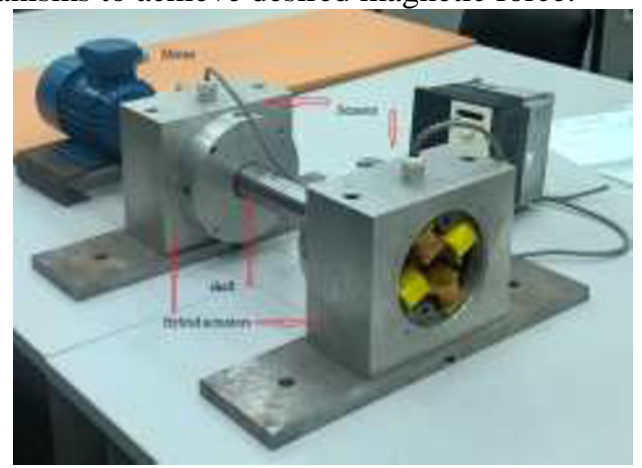

Figure 5. Balanced rotor system second prototype by strategies based on AMB.

According to get a sequence of steps to study the system,so it is summarized through flowchart of figure 6 . First, it must to be identified the system to get its physical parameters which are mass, damping, speed response, furthermore electrical parameters like resistance and inductance. Under this criterion, it must to be decided what mechanism or technique to use like ARMX or identification methodologies by polynomial analysis, etc. Next step is to test the controller due to AMB are unstable systems, and need a closed system analysis to get a control while identified the system.

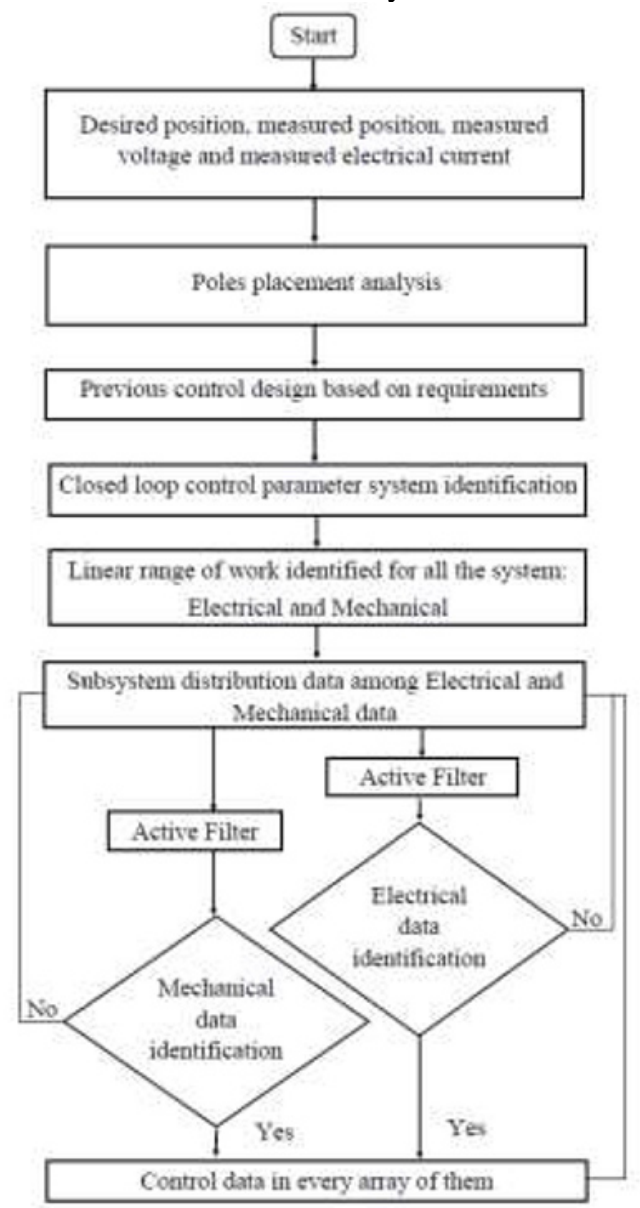

Figure 6. Proposed algorithm to control rotor position in unbalanced rotor, by AMB.
It was used a hybrid system identification (between frequency domain and time domain) inside the control algorithm, in order to improve adaptive control response for every linearized range of work.

In experiments, there was analyzed sensor position based in (Infrared) IR [7], due to response speed as dependence, furthermore actuator response time, computing time and response time of the system needed robust and fast sensors/ actuators. That task can be improved by Real Time analysis in algorithm design such as the processor to execute the main control system algorithm. Nevertheless, material science has achieved high advance nowadays in order to design new devices which can be used as sensors and it can provide robustness and fast response while system captures position information, this help is an advantage because of replace traditional sensors and to make simple algorithms.

\section{Physical parametres identification}

The proposal in this work is given for a prototype which is fixed with a DC motor, so its shaft is not balanced, therefor the AMB analyzed and suggested in this article can get a good control in addition to get a good performance for this system. The system can be analyzed by theoretical model such as physic laws description. The theoretical model can be proposed from equations that were showed in figure 2 .

Therefore, while it is given the dynamic equilibrium it is applied following equation analyzed from references, [4],[5], [6].

$$
\mathrm{F}_{\mathrm{Res}}=\mathrm{F}_{\mathrm{g}}+\mathrm{F}_{\mathrm{R}}+\mathrm{F}_{\mathrm{IL}_{\mathrm{i}}}+\mathrm{F}_{\mathrm{c}}
$$

where $F_{\text {Res }}$ is the resultant force in the system, $F$ is gravitational force, $\mathrm{F}_{\mathrm{R}}$ is Reaction Force, $F_{I L_{i}}$ is electromagnet force as resultant effect from every hybrid electromagnet, and $F_{c}$ is force because of rotation movement effect. Therefore, from which

$$
\mathrm{M} \frac{d^{2} z}{d t^{2}}=F g+F_{R}+F_{C}+K_{\mathrm{y}} y+K_{\mathrm{IL}} I L_{i}
$$

where the Matrix of mass is given by

$$
M=\left(\begin{array}{cccc}
m & 0 & \ldots & 0 \\
0 & m & \ldots & 0 \\
\vdots & \vdots & \vdots & \vdots \\
0 & 0 & \ldots & m
\end{array}\right)_{n \times m}
$$

The matrix that is composed for every stiffness coefficient is shown through following equation

$$
K_{Y}=\left(\begin{array}{cccc}
k_{y 1} & 0 & \ldots & 0 \\
0 & k_{y 2} & \ldots & 0 \\
\vdots & \vdots & \vdots & \vdots \\
0 & 0 & \ldots & k_{y n}
\end{array}\right)_{n x m}
$$

Furthermore, the matrix that is composed for every electrical current coefficient is shown by following equation 


$$
K_{i}=\left(\begin{array}{cccc}
k_{i 1} & 0 & \ldots & 0 \\
0 & k_{i 2} & \ldots & 0 \\
\vdots & \vdots & \vdots & \vdots \\
0 & 0 & \ldots & k_{i n}
\end{array}\right)_{n x m}
$$

Which means, the dynamical of the AMB system can be generalized for every displacement "Y", that

$$
Y=\left(\begin{array}{c}
Y_{1} \\
Y_{2} \\
\vdots \\
Y_{n}
\end{array}\right)
$$

In order to get the equilibrium of the system, the main control force is given by the Magnetic Force, it can be generalized for a hybrid condition, that means for an electromagnetic circuit in which the total magnetic field is produced because of permanent magnets and the electromagnets. This generalization enhance control response time, owing to the system can try to be in equilibrium while passive magnets are producing a magnetic force because of balancing the shaft and to compensate gravity force, reactions force and rotor movement inertia effect. Nevertheless, while the system loses the equilibrium, as a dependence of control algorithm, so the active electromagnet can look for the desired position; good advantage is given while the control response time is so short compared with response time of the system, because of to avoid heating transfer. That can be produced due to the magnetic circuit is working under conmutations, while it is up than $100 \mathrm{~Hz}$, so it can get behaviour of "Magnetic Furnace"; therefore, while control response time is short than response time of the system (even though disturbances).electrical power (as consequence to produce the electrical current to warrant the presence of magnetic field when active actuator is working) can be correlated with heating to be evacuated so fast because of the short control response time.

However, if in context when response time of the system is near the value of the control response time, so there is necessary to find not only programming strategics to reduce that difference; so, it is proposed to improve material of sensors and actuators. It means, as it researched nowadays, high advantages because of sensors/actuators based in nanostructures enhance robustness and response time owing to high ordered arrays of material distributed to elaborate the support of sensors/actuators, of course high dependence of material too.

In following equation is showen the lectrical current as dependence of Magnetic Field "B", Position " $r$ " and magnetic material properties "u", all as nonlinear behaviour through function "f".

$$
I_{i}=f\left(B_{i}, r_{i}, u_{i}\right)
$$

For electrical current as matrix form for equations worked.

$$
I=\left(\begin{array}{c}
I_{1} \\
I_{2} \\
\vdots \\
I_{n}
\end{array}\right)
$$

Furthermore, linearizing " $\mathrm{f}$ ", is possible to find the Magnetic Force as dependence of " $\mathrm{B}$ " and section area "A", that cross the magnetic field.

$$
F_{i}=\frac{B_{i}^{2} A_{i}}{2 \mu}
$$

As it was described above, heating transfer is necessary to analyse in these kind of systems too, in order to keep good control performance. That is a big misunderstanding to think AMB have not troubles with heating, that because of course by one side, heating produced through frictions of bearings is not given in this context due to levitation effect. However, when active control is working because active electromagnet actuator is working, that needs electrical current, and no matter commutations in order to reduce this electrical consumes, heating will be produced an that needs to be evacuated. Therefore, that is proposed a general heating transfer equation that is high correlated with geometrical characteristics of the system, as for this context by section area "A", length crossing for heating evacuation " $\mathrm{L}$ " and changing temperature " $T$ ", because of to evacuate heating "Q".

$$
\frac{d Q}{d r}=\frac{\alpha A}{L} T
$$

From which

$$
Q=\left(\begin{array}{c}
Q_{1} \\
Y_{2} \\
\vdots \\
Y_{n}
\end{array}\right)
$$

Also that cab be joined by this matrix

$$
\left(\begin{array}{cccc}
\frac{\alpha A_{1 \times 1}}{L_{1 \times 1}} & 0 & \ldots & 0 \\
0 & \frac{\alpha A_{2 x 1}}{L_{2 x 1}} & \ldots & 0 \\
\vdots & \vdots & \vdots & \vdots \\
0 & 0 & \ldots & \frac{\alpha A_{n \times n}}{L_{n x n}}
\end{array}\right)_{n x n}
$$

And from which matrix’s temperatura

$$
T=\left(\begin{array}{c}
T_{1} \\
T_{2} \\
\vdots \\
T_{n}
\end{array}\right)
$$

It is known that a usual System Identification methodology to identify physical parameters even though the main system is under disturbance, that is known as $\mathrm{ARX}$ as it is described in following equation, in which "A, B and C" are polynomials that depend of shift " $q$ ", owing to find correlation between output response " $y$ " as 
consequence of excitation signal " $u$ " and disturbance "e", all variable depende of time " $t$ ".

$$
A(q) y(t)=B(q) u(t)+C(q) e(t)
$$

So, it means

$$
\Theta=\left[a_{1}, a_{2}, \ldots, a_{n a}, b_{1}, b_{2}, \ldots, b_{n b}\right]^{T}
$$

Therefore

$$
r(t)=[-y(t-1), \ldots,-y(t-n a), u(t-n k), \ldots, u(t-
$$

Then, the physical parameters $\theta, \mathrm{r}$ can be obtained through

$$
\hat{y}\left(\frac{t}{\Theta}\right)=\Theta^{T} r(t)
$$

Other methodology usually in this context is given by a general polynomical analysis, that system identification criterion is sometimes known as "Modulating Functions" by some authors, which start from the general polynomial expression

$$
A \frac{d^{2} y}{d t^{2}}+B \frac{d y}{d t}+C y(t)=D R(t)
$$

In which $\mathrm{A}, \mathrm{B}, \mathrm{C}, \mathrm{D}$ are matrices that let to obtain physical

parameters of the system looking for to be identified, this Technique tries to analyse optimal solutions through verifying the least error as response of function " $R$ ". In context for this research, it was studied Least Mean Square in order to look for adaptive coefficients in order to optimize solution to get physical parameters. So while error "e" as difference from desired signal " $d$ " and measured adaptative signal " $\mathrm{S}$ " (or expected) as it is described by:

$$
e(n)_{y}=d(n)_{y}-S(n)_{y}
$$

For every " $\mathrm{S}$ " is composed by adaptive weight coefficients "w" correlated with input signal (measured or expected/simulated) "X".

$$
S(n)_{y i}=S(n)_{i} *\left[w^{T}(n)_{i} X(n)_{i}\right.
$$

For this reason, matrix of error is defined as

$$
e(n)_{y}=\left(\begin{array}{c}
e(n)_{1} \\
e(n)_{2} \\
\vdots \\
e(n)_{m}
\end{array}\right)
$$

And every component defines the Least Mean Square algorithm, that was worked in order to estimate transfer functions for the range of work 500 RPM until 1200 RPM and at maximal electrical current used for every electromagnet actuator was around 10A for random signal, in which for every steady state response was obtained (it is shown response for one of the electromagnet

$$
\frac{Y(S)}{\Delta I(S)}=\frac{12 e^{-6}}{50 S+1}
$$

Furthermore, it was analysed changes in shaft speed as disturbance

$$
\frac{Y(S)}{\Delta I(S)}=\frac{6 e^{-6}}{43 S+1}
$$

\section{Control position}

The control is proposed by an adaptative predictive model, in which high importance is given by a successfully system identification technique used to get the physical parameters of the system, even though, for this research was proposed system identification inside the main control algorithm as a strategy to get fast responses in front of disturbances, that was necessary to control the system during first identification, it because instability of the system (typical and AMB).

Therefore, the classic PID controller as it its shown in following equation in Laplace $(\mathrm{S})$ domain, in that " $\mathrm{K}_{\mathrm{P}}, \mathrm{K}_{\mathrm{I}}$, $\mathrm{K}_{\mathrm{d}}$ ” proportional constant, integral constant and derivative constant respectively.

$$
P I D(S)=K_{P}+\frac{K_{1}}{S}+K_{d} S
$$

The plant is divided in 2 different sections : an electrical section with a mechanical section that are described in following paragraphs.

\subsection{Control position dependent of electrical current as excitation variable}

In order to get the control, it is proposed a classical simple design as it shown in figure 4.1 , in which $\mathrm{I}(\mathrm{S})$ in the input electrical excitation signal, $\mathrm{Y}(\mathrm{s})$ is the position response, $\mathrm{C}(\mathrm{S})$ is the controller, $\mathrm{MS}(\mathrm{S})$ is the transfer function for the plant (that was used for mechanical part and electrical part of the system), and $S(S)$ is the transfer function for the sensor.

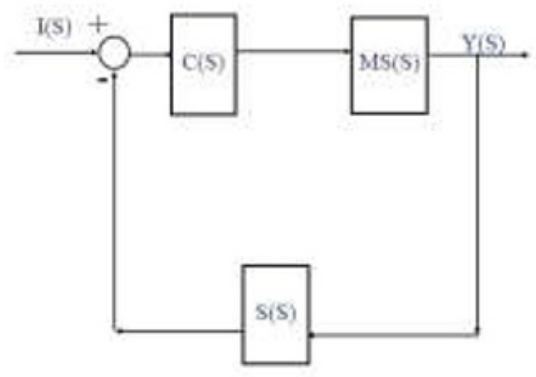

Fig. 7. Internal control system for identifications

Therefore, through algebra is obtained:

$$
(I(S)-S(S) Y(S)) C(S) P M(S)=Y(S)
$$

From which it is achieved following equation

$$
\frac{Y(S)}{I(S)}=\frac{C(S) M S(S)}{1+C(S) M S(S) S(S)}
$$

In last equation, it is considered own dynamic for actuators and sensors, nevertheless for this work it is considered an enough fast response in order to assign a gain behavior, so the transfer function it is considered as 
$\mathrm{S}(\mathrm{S})=\mathrm{K}_{\mathrm{s}}$ therefore in the characteristic equation for a classic PID model is reduced at following equation, in that " $\mathrm{m}$ " represented shaft's mass, $\mathrm{K}_{\mathrm{i}}$ and $\mathrm{K}_{\mathrm{y}}$ are electrical current coefficient and displacement coefficient.

$$
\left(K_{P}+\frac{K_{I}}{S}+K_{d} S\right)\left(\frac{K_{i}}{m S^{2}+K_{Y}}\right) K_{s}+1=0
$$

as reduction

$$
\left(m S^{2}+K_{z}\right)\left(K_{d} S^{2}+K_{p} S+K_{1}\right) K_{I} K_{S}+m S^{2}+K_{z}=0
$$

Working for a PI Controller, that means

$$
S^{3}+\frac{\left(1+K_{i} K_{Z} K_{Z}\right)}{K_{p} K_{i} K_{S}} S^{2}+\frac{\left(K_{Z} K_{p} K_{i} K_{S}\right)}{m K_{p} K_{i} K_{S}} S+\frac{K_{Z}\left(K_{I} K_{i} K_{S}+1\right)}{m K_{p} K_{i} K_{S}}=0
$$

While it is compared with third order polynomial model [6], it is necessary to recognize " $\omega_{0}$ " as the natural frequency assumed (expected for the system, " $e$ " as the capacity to overcome inertia (as damping effect) and " $\alpha$ " the coefficient which joins last both due to get comparison with main polynomial model.

$S^{3}+\omega_{0}(2 \epsilon+\alpha) S^{2}+\omega_{0}^{2}(1+2 \epsilon \alpha) S+\alpha \omega_{0}^{3}=0$ Otherwise, $K_{p} K_{I}$ and $\alpha$ can be obtained as functions of " $\omega_{0}$ " and " $\epsilon$ "

$$
\begin{gathered}
\frac{\left(1+K_{i} K_{y} K_{I}\right)}{K_{p} K_{i} K_{S}}=\omega_{0}(2 \epsilon+\alpha) \\
\frac{\left(K_{y} K_{p} K_{i} K_{S}\right)}{m K_{p} K_{i} K_{S}}=\omega_{0}{ }^{2}(1+2 \epsilon \alpha) \\
\frac{K_{y}\left(K_{I} K_{i} K_{S}+1\right)}{m K_{p} K_{i} K_{S}}=\alpha \omega_{0}{ }^{3}
\end{gathered}
$$

From equations (31), (32), and (33):

it means

$$
\alpha=\frac{1}{2 \epsilon}-\frac{K_{y}}{2 m \epsilon}
$$

$$
K_{i}=\frac{\left(m \omega_{0}-K_{y}\right) \alpha-2 K_{y} \epsilon}{K_{y} K_{I}\left(K_{S}-m \omega_{0}\right) \alpha-2 K_{I} K_{y} K_{S} \epsilon}
$$

For this reason, was possible to find $K_{i}$

$$
K_{i}=\frac{\left(m \omega_{0}-K_{z}\right) \frac{1-\frac{K_{z}}{m \omega_{0}^{2}}}{2 \epsilon}-2 K_{z} \epsilon}{K_{y} K_{I}\left(K_{S}-m \omega_{0}\right) \frac{1-\frac{K_{z}}{m \omega_{0}^{2}}}{2 \epsilon}+2 K_{I} K_{z} K_{S} \epsilon}
$$

Therefore

$$
K_{p}=\frac{K_{I} K_{y} K_{S}+\frac{K_{I}}{K_{i}}}{m K_{S} \omega_{0}^{3} \alpha}
$$

And finally, was possible to get the controller PI defined by its parameter proportional and integrative. This controller helps to find the right identification while system is stable

$$
K_{p}=K_{z} K_{I} K_{S}+K_{I}\left[1+\frac{4 \epsilon^{2}\left(K_{S} K_{I}-1\right)}{K_{I}\left(K_{S}-m \omega_{0}\right)\left(1-\frac{K_{I}}{m \omega_{0}^{2}}\right)-4 K_{S} \epsilon^{2}}\right]
$$

\subsection{Model Predictive Control Analysis}

Results above can get a control, nevertheless, not fast response as it could be obtained by a predictive model, either way such as by an Optimal Predictive Control described in following lines. The heating transition because of friction in bearings and calculated by analysis of friction coefficient special in mechanical bearing systems are not described in this work, due to this coefficient is null.
Notwithstanding, in this research was necessary to work with strategies of Model Predictive Control (MPC) joining both subsystems (mechanical and electrical) for the main algorithm, the strategy was an internal identification system while adaptive control coefficients/weights looked for the right control. Therefore, it is generalized for a nonlinear function " $\mathrm{f}$ " and internal variables " $x(t)$ " due to excitation " $u(t)$ " as function to time " $t$ ", [4],[5],[6].

$$
\frac{d x(t)}{d t}=f(x(t) u(t), \theta)
$$

By other side the general response " $y(t)$ " correlated with " $x(t)$ " and " $u(t)$ " through a nonlinear function " $h$ "

$$
y(t)=h(x(t), u(t), \theta)
$$

Also, while trajectory road " $\mathrm{R}_{\mathrm{S}}$ " as input excitation " $\mathrm{r}$ "

$$
R_{s}^{T}=(111 \ldots 11111) r\left(k_{i}\right)
$$

So, analyzing costing function "J", according to achieve the optimal desired position.

$$
J=\left(R_{S}-Y\right)^{T}\left(R_{S}-Y\right)+\Delta U^{T} R \Delta U
$$

In which this expected position is

$$
Y=H X\left(k_{i}\right)+\phi \Delta U
$$

Otherwise,

$$
\begin{array}{r}
J=\left(R_{S}-F X\left(k_{i}\right)\right)^{T}\left(R_{i}-F X\left(k_{i}\right)\right)-2 \Delta U^{T} \phi^{T}\left(R_{S}-\right. \\
\left.F X\left(k_{i}\right)\right)+\Delta U^{T}\left(\phi^{T} \phi+R\right) \Delta U
\end{array}
$$

In that, " $F "$ and " $\phi "$ are matrices that contain all physical parameters of the system (as joining matrices above regard identified result).

$$
\frac{\partial J}{\partial \Delta U}=-2 \phi^{T}\left(\left(R_{S}-F X\left(k_{i}\right)\right)+2\left(\phi^{T} \phi+R\right) \Delta U\right.
$$
So

$$
\frac{\partial J}{\partial \Delta U}=0
$$

From which, the optimal excitation signal in order to find the optimal response is given by

$$
\Delta U=\left(\phi^{T} \phi+R\right)^{-1} \phi^{T}\left(R_{S}-F X\left(k_{i}\right)\right)
$$

The total systems (mechanical and electrical subsystem) were controlled through following scheme of general cascade controller as shown in figure 8 , in which " $\mathrm{G}_{1}$ and $\mathrm{G}_{2}$ " are the controllers, furthermore, " $\mathrm{G}_{3}$ and $\mathrm{G}_{4}$ " are the subsystems of all the AMB model.

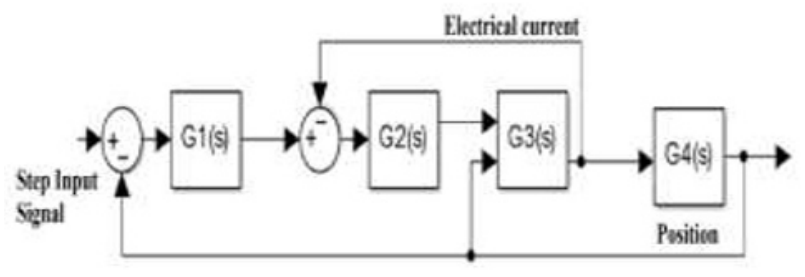

Fig. 8. Control cascade model for the total system. 
In figure 9 is depicted control result for the AMB prototype, in which is shown the position control for the simulation algorithm, that used the identification model parameters from the designed prototype. The control was achieved by Model Predictive Control, in which was achieved the best control for the polynomical identification model as part of the main controller.

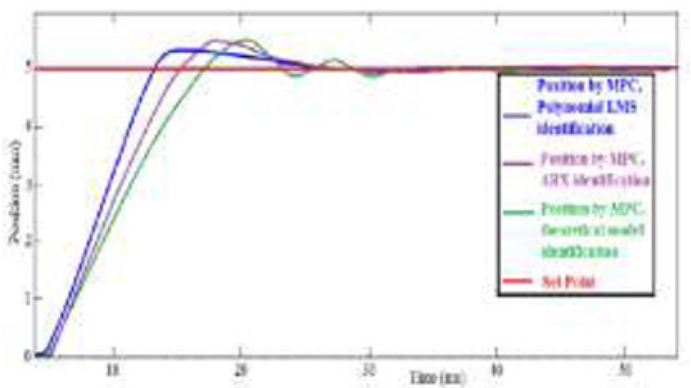

Fig. 9. Model Predictive Control simulation result (as dependence of identification methodologies) for the AMB prototype.

\subsection{Controllability and stability enhancement by sensor position based on nanostructures}

It was shown (mathematically analysed above) the response obtained by classical model through a sophisticated algorithm strategy; nevertheless, while it is used a robust and fast response sensor position, it implies more time to measure all information even though the system is under disturbances or imbalances, also the monitoring algorithm can be more simple and it increase memory space to make another complex tasks for the system. Otherwise, while actuators such as in this context is given by hybrid electromagnet/magnet actuator, it can get fast response compared with response time of the system (rotator RPM as for example) so, it enhance controllability and stability of the AMB.

In this research, it was discussed $\mathrm{AMB}$ bye passive mechanisms, from which was possible to find troubles under changes in frequency of the system, therefore that could be improved by active mechanisms (electromagnet actuator) for this reason it was proposed hybrid strategies to build the actuator (hybrid among passive and active). Notwithstanding, even though this suggested design could enhance heating evacuation because of short time to get action by the active control, it means all system can be under equilibrium by the passive actuator and under uncontrol, the active actuator (part of the hybrid) can try to get the control of the AMB.

Furthermore, in this work it is suggested to use sensors and actuators based in nanostructures due to "Robustness and so short response time" for specific cases when response time of the system (high RPM applications) can produce unsuitability even though the system could be a hybrid and controlled by good strategies. Therefore, sensors based in nanostructures can achieve fast information because of good molecular organization and similar context for hybrid actuators in which magnet components are based in nanostructures, owing to good molecular organization can transmit as a better way the magnetic field in order to produce fast controlled electromagnetic force.

In figure 10 is shown the comparison between the MPC result obtained above, with the MPC used while the system is designed (emulated) by sensor/actuators based in nanostructures. As it was expected, nanostructures get a good performance as a consequence also in the control. Analyzing [8], [9], [10], [11], it was studied material proposals for many researchers, their geometry organization due to high ordered array, also their different responses as dependence of material and geometry of nanostructures from which were estimated static and dynamic behaviour in order to emulate transfer functions for similar linear range domain with AMB's sensor /actuator range domain and composition.

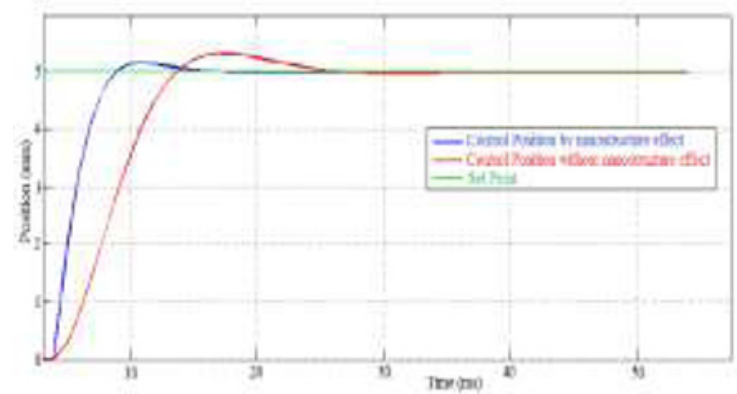

Fig. 10. AMB control comparison among control with and without nanostructure effects.

\section{Conclusions}

This work shows a good control position performance for a generalized AMB system, while it is find the equilibrium among desired trajectory, system stability and avoiding response for different disturbances. Notwithstanding, disadvantages of these algorithms are given by not enough memory space to execute them, due to computing time is short compared with rotor displacement response time, although while it could be possible to execute the control algorithm through a Real Time operating system in order to get desired response.

By other side, it is shown by simulations a fast and robust position sensor (based in nanostructures) that can replace to traditional position sensor in required range of work, besides, either way this position sensors based on AAO templates let to get more free memory space in controllers, which can be used to solve more complicated task for the system such as an improved artificial intelligence.

It was proposed a generalized model for AMB, which can be particularized to different prototypes as a consequence of selecting physical parameters in the main mathematical model of the AMB system, furthermore it is tested enhanced results in the control position system.

\section{Suggestions}

It is proposed that position sensor and hybrid electromagnet actuators based on nanostructures can provide robustness fast response which is desired for $\mathrm{AMB}$, due to do not lose stabillity and controllability 
while rotor gets high speed compared with its own nature frequency. That information, obtained by simulations and mathematical analysis in this work, is suggested to be verified by experiments for that is necessary to replace traditional gap/position sensors and traditional hybrid electromagnet actuators by sensors/actuators based in nanostructures.

The author wishes to thank to Mr. César José Leclere Mota and Mr. Bruno Sebastián Miranda Quispe because of their support in experimental tests.

\section{References}

1. Krause, Paul; Wasynczuk, Oleg (1994). Analysis of Electric Machinery. IEEE PRESS Marketing.

2. Zawoysky, Ronald; Tornoroos, Karl. GE Generator Rotor Design, Operational Issues, and Refurbishment Options. GE Power Systems.

3. ABB Motors and Generators (2012). Generators for Wind Power, Proven generator - reliable power.

4. Ljung, Lennart (2014). System Identification Toolbox, Getting Starting Guide. www.mathworks.com.
5. Sae-Wan Kim (2017). Easy-to-Fabricate and HighSensitivity LSPR Type Specific Protein Detection Sensor Using AAO Nano-Pore Size Control.

6. Karl Johan Astrom (2002). Control System Design

7. General Purpose Type Distance Measuring Sensors, Technical information. SHARP GP2Y0A21YK/GP2Y0D21YK.

8. F N Werfel(2012). Superconductor bearings, flywheels and transportation

9. Il Seo (2009). Assembly of Colloidal Nanoparticles into Anodic Aluminum Oxide Templates by DipCoating Process.

10. Nanolike, temperature nanosensor. www.sensortest.de/ausstellerbereich/upload/mnpdf/en/ Nanoliketemperaturenanosensors15.pdf.

11. Yong Lei (2006). High ordered nanostructures with tunable size, shape and properties: A new way to surface nanopattering using ultrathin alumina masks. 